\title{
Integral Invariants for Image Enhancement
}

\author{
Faraz Janan, Sir Michael Brady
}

\begin{abstract}
Medical images pose a major challenge for image analysis: often they have poor signal-to-noise, necessitating smoothing; yet such smoothing needs to preserve the boundaries of regions of interest and small features such as mammogram microcalcifications. We show how circular integral invariants (II) may be adapted for feature-preserving smoothing to facilitate segmentation. Though II is isotropic, we show that it leads to considerably less feature deterioration than Gaussian blurring and it improves segmentation of regions of interest as compared to anisotropic diffusion, particularly for hierarchical contour based segmentation methods.
\end{abstract}

\section{I TRODUCTIO}

Medical images pose a major challenge for image analysis: often they have poor signal-to-noise, necessitating smoothing; yet such smoothing needs to preserve the boundaries of regions of interest and small features such as microcalcifications so that they can be segmented accurately. Image smoothing techniques are generally divided into two broad classes: isotropic and anisotropic. The former, typified by Gaussian scale-space analysis, operate at each pixel (or voxel in 3D) uniformly in all directions; the latter operate preferentially in a particular direction determined by the local image content (typically estimated from the image gradient). Though there is an extensive literature on anisotropic methods, few isotropic methods are popular in practice. Typically, Gaussian filtering is used to suppress noise by diffusing the image content [4]. Applying a Gaussian filter essentially amounts to a low pass filtering the image, assuming that the high frequency component is of little or no consequence. This is clearly not true at edges, and predominantly in medical imaging applications, such as in the case of mammography, where the high frequency content - microcalcifications, curvilinear (ductal) structures, and spicules of masses are of major clinical significance. The challenge therefore is to remove noise so as to enhance the image for human or machine analysis while not suppressing important high frequency information. We propose the use of integral invariants as smoothing kernels to enhance images for segmentation and shape analysis. We first show that II is a linear filtering operation that diffuses noise isotropically yet preserves high frequency information content substantially more effectively than Gaussian smoothing.

Faraz Janan is a DPhil Engineering Science candidate at the Institute of Bio-Medical Engineering (IBME), University of Oxford. Phone: +44 (0) 7550505969, e-mail: faraz.janan@some.ox.ac.uk.

Sir Michael Brady FRS FREng FMedSci, is Professor of Oncology at the Department of Oncology, University of Oxford UK.
Figure 1 shows the Fourier transform of a mammogram and compares its Gaussian and integral invariant diffused versions. It can be seen clearly that II preserves high frequency components as compared to Gaussian filtering. Second, we study the trade-off between image and shape quality as a result of anisotropic diffusion and the benefits that II diffusion may offers in his regard. We compare II to Perona-Malik (PM) anisotropic diffusion [3, 11]. Evidently, the choice between these two depends upon the specific application; however, we find that for mammograms and for the segmentation algorithm that we use, II gives better results. By changing the radius $r$, II can operate at a succession of scales whereas the action of PM depends less transparently upon the number of iterations $n$ over which it is applied as well as the size of the image gradient.

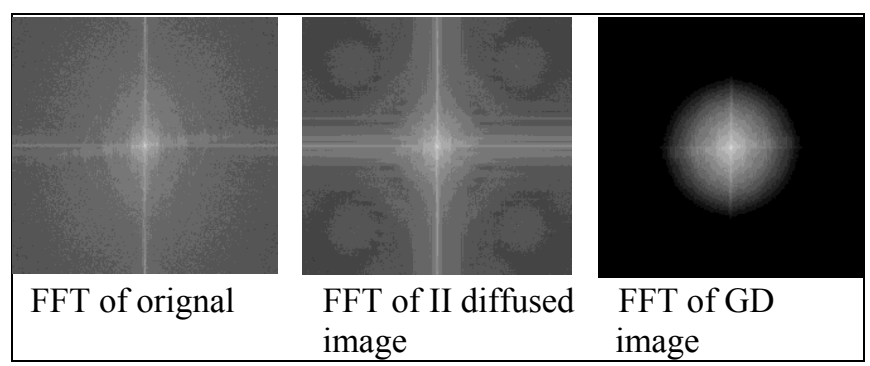

Figure 1: FFT of a mammogram and its diffused versions

\section{U BOU DED I TEGRAL I VATIA TS}

Manay et.al [2] define (circular area) integral invariants by considering a disc $B_{r}(p)$ of radius $r$ applied to every point $p$ of a closed contour $C$, the characteristic function is then given by,

$$
\chi\left(B_{r}(p), C\right)(x)=\left\{\begin{array}{c}
1 \text { if } x \in\left\{B_{r}(p) \cap \dot{\mathrm{C}}\right\} \\
0 \text { otherwise }
\end{array}\right.
$$

Where $\dot{C}$ is the interior of the curve $C$. The local integral area $I_{r}(C)$ of the curve $\mathrm{C}$ is given by the function $I_{r}(p)$ at every point $p \in C$ with integral kernel $\chi$ as follows:

$$
I_{r}(p)=\int_{\Omega} \chi\left(B_{r}(p), C\right)(x) d x
$$

Where $\Omega$ is the domain of the curve $C$.

IIs have been shown to be effective for shape matching and for local region matching because they provide a natural multi-scale description of a shape. IIs have the expressive power to encode a shape; they resemble the representation of a shape using curvature functions [1]. However, and 
crucially, compared to differential invariants such as curvature, IIs are substantially more robust to noise [5].

A variation of circular II, referred here as unbounded integral invariant is used. Instead of imposing a constraint on the integration area to overlap with a particular shape; we integrate over the complete area of the kernel that overlaps with any points inside the image. Hence, $\chi\left(B_{r}(p), C\right)$ is actually the area of the integration kernel.

\section{RESULTS A D OBSERVATIO S}

Various aspects of II are analysed and compared to PM and to Gaussian diffusion. Note that we do not dispute the superiority of PM when used with a small gradient and a suitably large number of iterations in preserving crisp clear edges for being anisotropic. PM works similar to the process of creating a scale space, where an image is convolved with a family of convolution kernels that increases in width to form a scale space $[11,12]$. However, PM is non-linear and spatially variant in its application over an image. It computes a filter shape that is elongated and has an orientation that is adapted for each point in the image (at each scale). It smooths a region within its boundary that are significant edges or lines of a certain strength, and not across the boundary. It diffuses the gradients under a given threshold value with a low contrast and enhances the opposite with a high contrast.

\section{A. Enhancement of image features}

We have found that II enhances the boundaries of image features such as edges and lines by quantizing them into more intensity levels as compared to PM. This generates a contour map for a shape that has a substantially higher number of contours surrounding the shape. This in turn aids contour-based hierarchical segmentation algorithms. Figure 2 illustrates this, an image diffused by II has the boundaries of the shapes enhanced, considerably more so than in comparison to PM.
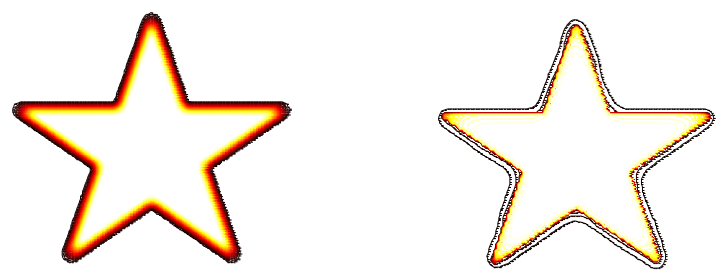

Figure 2: Contour models of II (left) \& PM (right) diffused phantom shape

Previously, integral invariants have also been used on mammograms for local region matching of segmented shapes [13]. Here, we have applied the method to a set of mammograms, for example the one with a mass shown in Figure 3. The contour model of the mammogram smoothed by II has more dense contours surrounding the mass and so appears darker in the false colour map. This facilitates the automatic detection and segmentation of the mass.

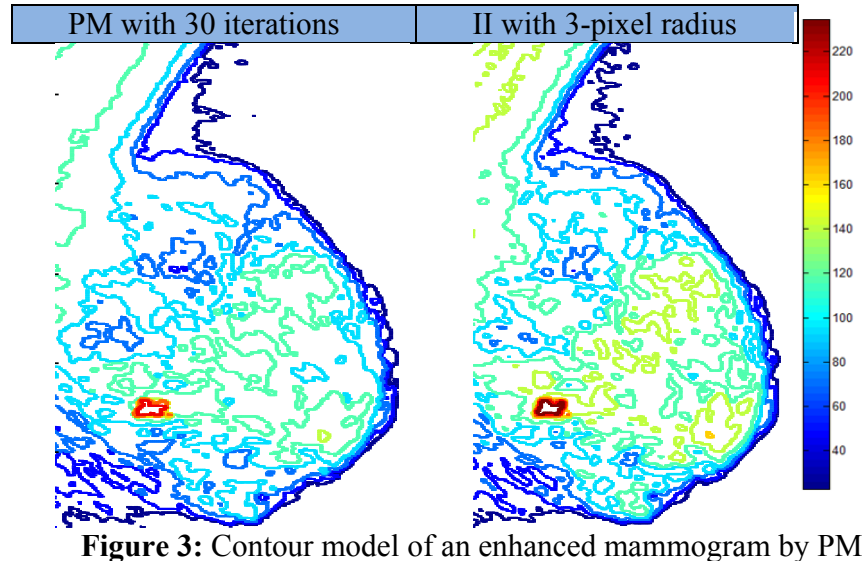

(left) and II (right). The later has enhanced region.

\section{B. Noise Suppression}

We have assessed II for noise suppression against PM. Here, we do not rely upon the SNR of the diffused phantom for comparison, as II is more aggressive in smoothing image details; it gives a lower SNR than PM in all cases. 'Salt and pepper' and 'multiplicative uniform' noise models are added to the phantom image. Figure 4 shows the noise suppression results. A slight asymmetry may be noticed in the diffused versions of II, which increases with increasing scale. This is because one end of the star shape is convex, which collapses on itself as scale increases. The other end is concave, which expands downward as a result of diffusion. Together, these shift the intensity pattern from right to left, making it asymmetric to the original profile. (a)

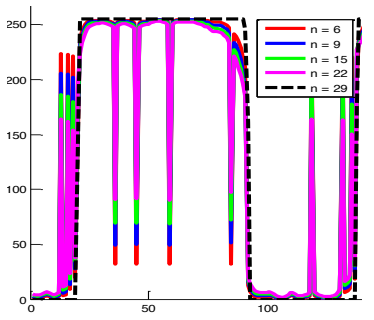

(b)

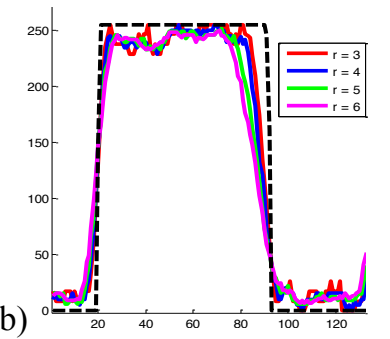

Figure 4: Scale space comparison of II and PM diffused section of intensity profile across the star shape in Figure 2. Here, phantom is acquired by added multiplicative Gaussian noise and generated after applying salt \& pepper noise. PM diffused intensity profile is on the left, while II is on the right. $n$ is the number of iterations for Perona-Malik, whereas, $r$ is the radius of integral invariant kernel.

In Figure 5, a mammogram is smoothed to remove noise. The original mammogram contains a layer of dusty noise (shown in blue), shown in (a) at the right end of the image that surrounds the mammogram concavely. In (b) PM is shown to have reduced this artefact. In (c), II has eliminated the noisy layer completely while the breast boundary is retained.

We observe that PM clusters high intensities at the borders of the image and induces an unwanted artefact that may give rise to new image structures. This may be problematic in applications where image borders contain 
important information. II does not induce such artefacts, as shown in Figure 6.

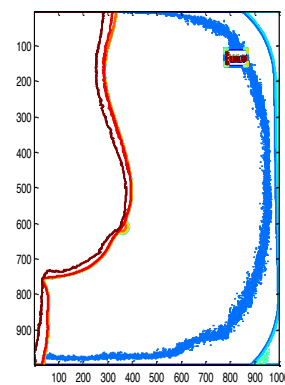

(a)

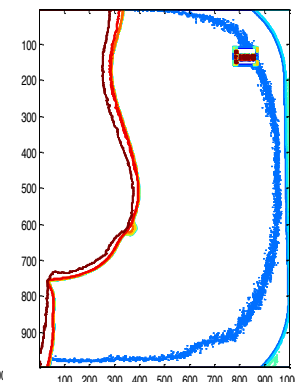

(b)

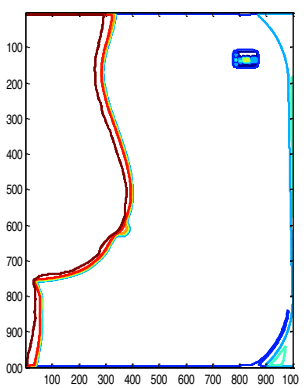

(c)
Figure 5: (a) A contour map of a noisy DICOM mammogram. (b) smoothed by PM, (c) smoothed by II.
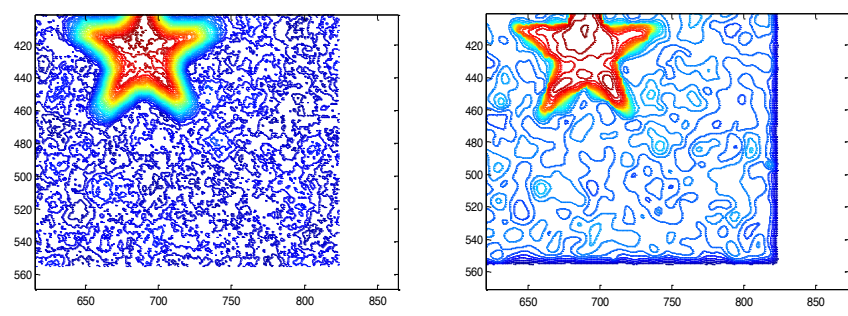

Figure 6: Contour models show the effects of II (left) and PM (right) on image boundary

\section{Efficiency with respect to segmentation results}

Image smoothing is known to reduce the number of false positives and negatives in image segmentation applications. For this reason, we evaluated II against other well-known noise suppression techniques: speckle reducing anisotropic diffusion (SRAD) $[6,8]$; total variation denoising using the Sobolev method (TV) [7, 8]; and PM. The algorithm has been applied to 10 temporal pairs of volumetric density maps for the mammograms. The maps were generated by Volpara [9].

\section{Performance of noise adjustment methods with respect to segmentation results for density mammograms}

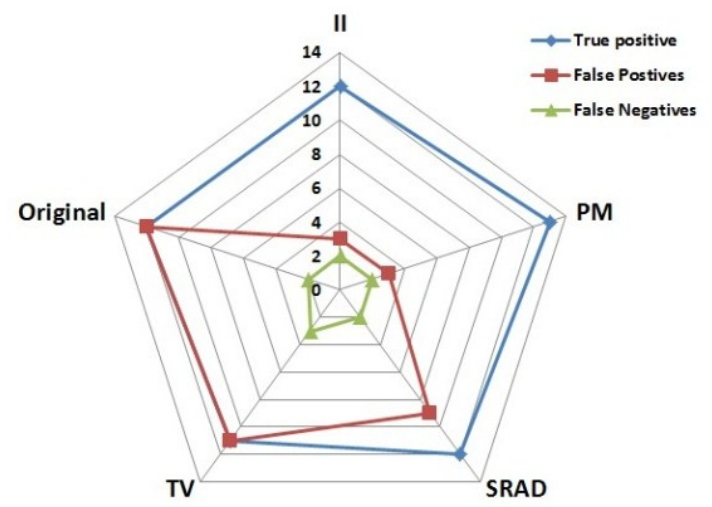

Figure 7: II and PM gives the lowest number of false positives and false negatives, and performs well for finding true positives
A variation of a topographic segmentation algorithm based on iso-contours [10] is used to delineate regions of interest. A total of 14 lesions were selected as ground truth. II and PM perform equally well in reducing the number of false positives and negatives, where the latter performs best in detecting true positives. For one mammogram, the lesion was not detected by either of the methods. Results are summarized in Figure 7.

\section{Shape Deterioration by smoothing kernels}

This section analyses the effect of II and Gaussian diffusion (GD) on shape deterioration and intensity flow. Object deterioration as a result of intensity diffusion depends upon two factors. The first is the ratio of size of the object to the size of the diffusion kernel. The second is the overall shape of the object.

There are two ways to approach shape deterioration at multiple scales. If a set of kernels of varying sizes is applied to a shape, we will get a set of new shape boundaries (shape dilation), as well as flow of intensities through the original boundaries as a result of diffusion, thus creating a scale space. For convenience, we call these boundary scale space (BSS) and intensity scale space (ISS) as they refer to the deterioration of objects in terms of boundary and its diffused intensity, respectively. In the case of BSS, we have the outer boundary of diffusion that will actually expand the shape area and the inner boundary that will show the deterioration and may diminish the structure. Figure 8 shows the boundaries of a diffused spiculated mass phantom at a certain scale. It is clear that II leads to less deterioration of shapes as compared to GD at corresponding scales. It can be seen that integral invariants retain shape information more accurately and resist deterioration in contrast to GD. In both of these examples, II better maintains the overall shape signature in terms of outer boundary and resist deterioration as for the inner boundary, as compared to GD. Figure 9 shows an example of a pairs of automatically segmented temporal mammograms density maps, enhanced by II. It can be seen that II has not deteriorated the regions of interest (delineated in blue) despite their small size and have suppressed any false positives.

\section{DISSCUSSIO}

We have evaluated II as an image enhancement method and compared it to PM anisotropic diffusion and to Gaussian smoothing. We have shown that it is a reasonable trade-off between the two, where in some applications it could be preferred over Perona-Malik with a number of advantages as explained. We also evaluated it over mammograms as a preprocessing method to reduce the number of false positives and negatives and found it almost effective as Perona-Malik, though computational more efficient. For Gaussian smoothing, it leads to substantially less deterioration of important shape features. It preserves high frequencies to a larger extent and is less aggressive in deteriorating shapes as compared to Gaussian filter. Particularly in shape analysis 
application that requires shape description [2], it eliminates the need for a separate noise removal filter, as well as enhancing image features for intensity based segmentation methods.

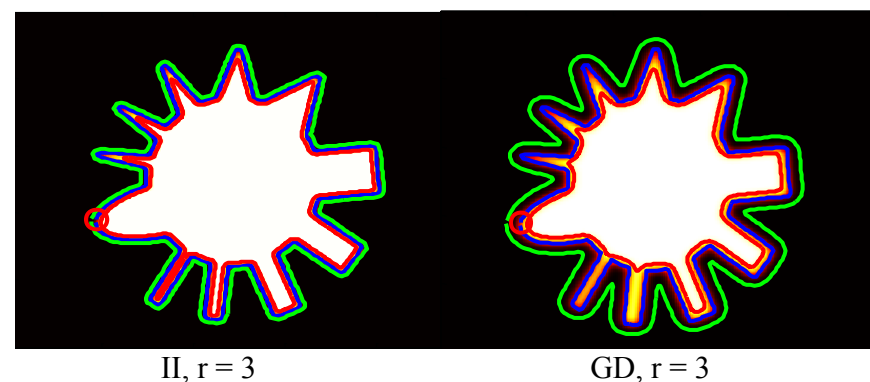

Figure 8: Diffusion of the image by II and GD at corresponding scales. Blue line is the original shape boundary, green and red are outer and inner boundaries as a result of diffusion. II shows an obvious advantage over GD in terms of shape determination

\section{REFERE CES}

[1] F. Mokhtarian and A. K. Mackworth, "Scale-Based Description and Recognition of Planar Curves and Two dimensional Shapes", IEEE Trans. PAMI., vol. PAMI-8, pp. 3443, 1986.

[2] S. Manay, D. Cremers, B.-woo Hong, A. J. Y. Jr, and S. Soatto, "Integral Invariants for Shape Matching," PAMI, October, vol. 28, no. 10, pp. 1602-1618, 2006.

[3] Perona, P., \& Malik, J. (1990). "Scale-space and edge detection using anisotropic diffusion". IEEE Transactions on Pattern Analysis and Machine Intelligence, 12(7), 629-639. doi: 10.1109/34.56205.

[4] A. P. Witkin, "Scale-space filtering", in Proc. 8th Int. Joint Conf. Art. Intell., (Karlsruhe, West Germany), pp. 1019(1022), Aug. 1983.

[5] H. Pottmann and J. Wallner, "Integral invariants for robust geometry processing," Computer Aided Geometric Design, vol. 26, pp-37-60, 2009.

[6] Y. Yu and S. T. Acton, "Speckle reducing anisotropic diffusion.," IEEE transactions on image processing: a publication of the IEEE Signal Processing Society, vol. 11, no. 11 , pp. 1260-70, Jan. 2002.

[7] S. Monica, "Nonlinear total variation based noise removal algorithms", Physica-D, vol. 60, pp. 259-268, 1992.

[8] http://www.ceremade.dauphine.fr/ peyre/numerical-tour/

[9] R. Highnam, S. Brady, M. Yaffe, and N. Karssemeijer, "Robust Breast CompositionMeasurement-Volpara" TM Digital, 2010.

[10] B.-woo Hong and M. Brady, "Segmentation of mammograms in topographic approach," International Conference on Visual Information Engineering (VIE 2003). Ideas, Applications, Experience, pp. 157-160, 2003.

[11] J. Weickert, Anisotropic diffusion in image processing. 1998.

[12] T. Lindeberg, "Scale Selection Properties of Generalized Scale-Space Interest Point Detectors," Journal of Mathematical Imaging and Vision, Sep. 2012.

[13] F. Janan and S. Brady, "Region matching in the temporal study of mammograms using integral invariant scale-space," Breast Imaging, pp. 173-180, 2012.

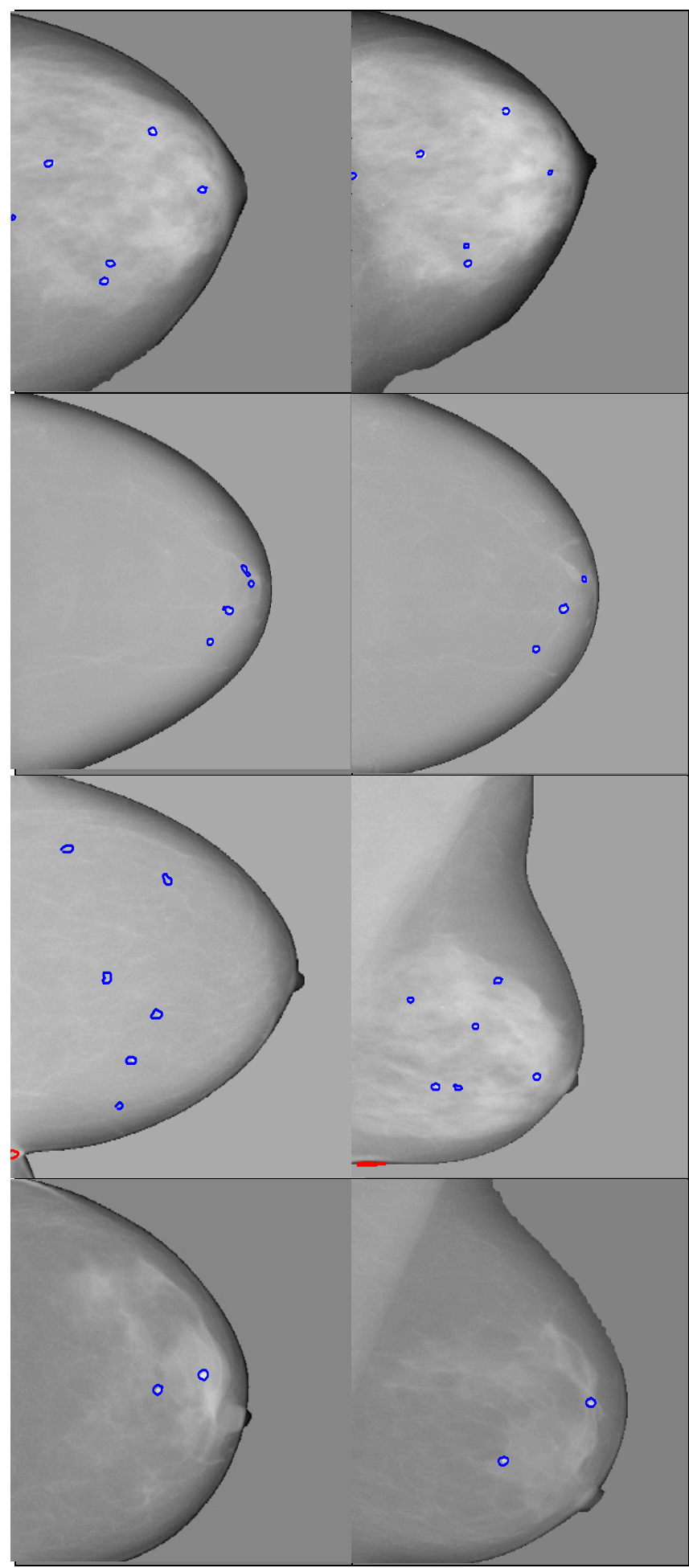

Figure 9: Automatic Segmentation of Integral Invariants enhanced temporal pairs of mammogram density maps created by Volpara [9] 